\title{
Lesão Neurológica em Cirurgia Cardíaca: Aspectos Fisiopatológicos *
}

\section{Pathophysiology of Neurological Injuries during Heart Surgery}

\author{
Rólison Gustavo Bravo Lelis ${ }^{1}$; José Otávio Costa Auler Júnior, TSA ${ }^{2}$
}

\author{
RESUMO \\ Lelis RGB, Auler Jr JOC - Lesão Neurológica em Cirurgia Car- \\ díaca: Aspectos Fisiopatológicos
}

JUSTIFICATIVA E OBJETIVOS: Em virtude de as lesões neurológicas ocuparem um papel importante no contexto das complicações pós-operatórias, quando comparadas às demais, o presente estudo procurou discutir os principais fatores envolvidos na lesão neurológica peri-operatória em cirurgia cardíaca, as intervenções que buscam diminuir a incidência de lesões neurológicas, enfocando de maneira simples, e a provável gênese genética em tais lesões encefálicas.

CONTEÚDO: Este trabalho contém a revisão de artigos que enfocam a fisiopatologia das lesões neurológicas relacionadas a procedimentos cardíacos, sua possível origem genética, bem como uma proposta para sua prevenção.

CONCLUSÕES: Muito tem se falado das disfunções cognitivas de pacientes após cirurgia cardíaca, como a revascularização do miocárdio que é motivo do aumento da morbimortalidade observada atualmente e fator de maior tempo de hospitalização. É fato que já houve grandes avanços nessa área a fim de diminuir esses índices, prevendo-se ainda outros que visam a profilaxia de lesões neurológicas.

Unitermos: CIRURGIA, Cardíaca: revascularização do miocárdio; COMPLICAÇÕES, Neurológica: lesão

\author{
SUMMARY \\ Lelis RGB, Auler Jr JOC - Pathophysiology of Neurological Inju- \\ ries during Heart Surgery
}

BACKGROUND AND OBJECTIVES: Since neurological injuries are very concerning and important postoperative complications following cardiac surgeries, the purpose of this study was to discuss the pathophysiology of these injuries, what measures can be taken to decrease their incidence and the possible genetic origin of such brain injuries.

CONTENTS: This study is a review of papers which address the pathophysiology of heart surgery-related neurological injuries and their possible genetic origin, as well as some proposals for their prevention.

CONCLUSIONS: A lot has been said about cognitive function disorders after heart surgeries, such as myocardial revascularization, which are present causes of increased morbidity and mortality, in addition to longer hospital stay. Major advances to decrease such rates have been observed in this field, and others are still expected aiming at neurological injuries prevention.

Key Words: COMPLICATIONS, Neurologic: injuries; SURGERY, Cardiac: myocardial revascularization

\section{INTRODUÇÃO}

$\mathrm{O}$ avanço tecnológico das últimas décadas tem levado à diminuição da morbimortalidade em cirurgias cardíacas. Porém, as complicações neurológicas ainda representam importante causa de morbidade no período pós-operatório, respondendo por grande parcela de óbitos. Há cinco décadas, a circulação extracorpórea (CEC) foi introduzida nas cirurgias e desde então, começaram os relatos que certos pacientes desenvolviam algum tipo de seqüela

\footnotetext{
* Recebido do (Received from) Serviço de Anestesiologia e Terapia Intensiva Cirúrgica do Instituto do Coração da Faculdade de Medicina da Universidade de São Paulo - InCor (FMUSP), São Paulo, SP

1. Graduando de Medicina da FMUSP. Bolsista de Iniciação Científica pela FAPESP (Processo $n^{\circ} 02 / 06790-9$ )

2. Professor Titular da Disciplina de Anestesiologia, Departamento de Cirurgia da FMUSP
}

Apresentado (Submitted) em 25 de julho de 2003

Aceito (Accepted) para publicação em 26 de novembro de 2003

Endereço para correspondência (Correspondence to)

Prof. Dr. José Otávio Costa Auler Júnior

Av. Dr. Enéas de Carvalho Aguiar, 44 Cerqueira César

05403-000 São Paulo, SP

E-mail: auler@hcnet.usp.br

(C) Sociedade Brasileira de Anestesiologia, 2004 neurológica. A partir daí, as seqüelas neurológicas e a própria CEC têm sido alvo de intensas pesquisas. Acredita-se que a incidência dessas complicações, de diferentes graus, alcance até $75 \%$ dos pacientes ${ }^{1}$, complicações essas que podem variar desde alterações psicológicas transitórias, como deficits de atenção e memória, até eventos mais graves, como acidente vascular encefálico (AVE) extensos. Atualmente, sabe-se que essas complicações, após a cirurgia cardíaca, podem englobar diversas áreas do encéfalo e manifestar-se de diferentes formas clínicas.

Inúmeros fatores agem de forma diferente na gênese da lesão neurológica peri-operatória em cirurgia cardíaca. Dentre eles, podem ser citados a idade avançada, a presença de doença encefálica-vascular prévia e de doença ateromatosa, o diabete melito, os procedimentos intracardíacos, a alteração na temperatura corporal durante a CEC, e talvez uma predisposição genética entre outros.

O objetivo desta revisão é discutir os principais fatores envolvidos na lesão neurológica peri-operatória em cirurgia cardíaca e as intervenções que buscam diminuir sua incidência enfocando, de maneira simples, a provável gênese genética em tais lesões encefálicas. 


\section{INCIDÊNCIA E ASPECTOS FISIOPATOLÓGICOS}

Pode-se observar que a incidência de complicações neurológicas no pós-operatório de cirurgia cardíaca varia bastante na literatura especializada. A discrepância dos resultados é atribuída ao desenho do estudo, se prospectivo ou retrospectivo, às operações cardíacas com a cavidade aberta ou fechada, à presença de comorbidades e o método utilizado para avaliar o tipo e grau das disfunções neurológicas. As pesquisas relacionadas à incidência e disfunção neurológica no pós-operatório estão principalmente direcionadas à cirurgia de revascularização do miocárdio. Isto se deve ao fato de representar o maior contingente dentre as operações cardíacas, explicando o porquê de a maioria dos estudos referir-se a este tipo de operação. Embora haja divergência quanto ao método, a incidência de complicações neurológicas varia entre $0,4 \%$ e $5,4 \%{ }^{1}$ nos diferentes centros.

Mesmo reconhecendo-se ser a circulação extracorpórea o principal fator determinante das complicações neurológicas em cirurgia cardíaca, sempre houve alguma dúvida quanto ao potencial efeito nocivo sobre o sistema nervoso central do ato anestésico-cirúrgico. Para verificar este fato, estudo europeu $^{2}$, avaliou a função encefálica de 1.218 pacientes idosos submetidos à cirurgias não-cardíacas. Exames neurocognitivos compostos por questionários simples, com perguntas diretas sobre nome, data do nascimento, data atual, profissão, endereço, dados sobre os familiares, foram aplicados em três períodos, antes da cirurgia, na alta hospitalare após três meses. Observou-se que havia uma incidência relativamente elevada de disfunção cognitiva pós-operatória (26\%), traduzida por confusões com datas e fatos, bem como lapsos de memória que persistiram por até três meses em $10 \%$ dos pacientes estudados ${ }^{2}$. O estudo procurou imputar ao ato anestésico-cirúrgico a responsabilidade por esta perda de funções cognitivas, na ausência de circulação extracorpórea. Shaw e col. ${ }^{3}$, nesta mesma linha de investigação, também utilizando testes neurocognitivos pautados em questionários de perguntas e respostas simples, compararam dois grupos de pacientes: um submetido à cirurgia de revascularização do miocárdio com auxílio de circulação extracorpórea e outro, à cirurgia vascular periférica. Os resultados encontram-se na tabela I, denotando-se maior incidência de disfunção cognitiva no primeiro grupo. O que pode se depreender destas e de outras investigações é que, certamente, a circulação extracorpórea constitui um fator predisponente para disfunções cognitivas, mas que estas também estão presentes em cirurgias não-cardíacas, principalmente em pacientes com antecedentes de doença vascular arterial. O impacto das alterações cognitivas sobre a morbimortalidade dos pacientes submetidos à cirurgia cardíaca com circulação extracorpórea, não é muito intenso. O mesmo não se pode dizer de intercorrências mais sérias, uma vez que lesões definidas genericamente como acidentes vasculares encefálicos (AVE) podem conduzir a estado de coma, a óbito ou a seqüelas permanentes. Sempre houve uma certa confusão na literatura no que tange a denominação, classificação e fisiopatologia destes eventos neurológicos mais graves.
Tabela I - Complicações Neurológicas na Cirurgia Cardíaca Comparada à Cirurgia Vascular Periférica ${ }^{3}$

\begin{tabular}{lcc}
\hline Gravidade do Deficit & $\begin{array}{c}\text { Revascularização do } \\
\text { Miocárdio } \\
(\mathrm{n}=298)\end{array}$ & $\begin{array}{c}\text { Controle } \\
(\mathrm{n}=48)\end{array}$ \\
\hline Leve (deficit em 1 ou 2 testes) & $164(55 \%)$ & $15(31 \%)$ \\
Moderado (deficit em 3 ou 4 testes) & $57(19 \%)$ & 0 \\
Grave (deficit em $\geq 5$ testes) & $14(4,7 \%)$ & 0 \\
\hline
\end{tabular}

Coube a um grupo de pesquisadores, tendo Roach e col. ${ }^{4}$ à frente, investigando ampla série de pacientes, apresentar uma proposta de classificação. A par da extensa casuística revisada, Roach e col. propuseram uma classificação, delineada no quadro I. Utilizando a classificação proposta por estes pesquisadores ${ }^{4}$, Wolman e col. ${ }^{5}$ avaliaram um grupo de pacientes submetidos à revascularização do miocárdio, classificando as intercorrências neurológicas em: tipo I, definidas como lesão focal, com presença de estupor ou coma; e tipo II com deterioração da função intelectual, deficit de memória ou convulsões ${ }^{5}$.

Quadro I - Proposta de Classificação de Eventos Neurológi$\cos ^{5}$

Morte encefálica, acidente vascular encefálico não-fatais e

Tipo I ataques isquêmicos transitórios (fatores predisponentes: aterosclerose aórtica, doença neurológica prévia, idade)

Diminuição da capacidade intelectual, (fatores predisponentes:

Tipo II idade, hipertensão, arterial, doença pulmonar e consumo excessivo de álcool)

O resultado deste estudo demonstrou que 6,1\% dos pacientes apresentaram alguma complicação neurológica, sendo que $3,1 \%$ deles acusaram alterações do tipo le $3 \%$ do tipo $\|^{5}$. Nesta mesma investigação relacionada à cirurgia cardíaca, Wolman e col. ${ }^{5}$ mostraram que, paralelamente à técnica cirúrgica, a idade também seria um fator de risco para complicações neurológicas. Pacientes com idade igual ou maior que 70 anos apresentaram, em média, $4 \%$ a 9\% de incidência de AVE ou coma após a operação, diferentemente dos pacientes com idade abaixo dos 70 anos, que apresentaram incidência de $1 \%$.

Outros mecanismos que possam explicar essas complicações também têm sido exaustivamente estudados. A própria CEC tem sido considerada como a causa principal das disfunções cognitivas mais sérias no pós-operatório. No entanto, estudo realizado por Taggart e col. ${ }^{6}$, a fim de determinar o real impacto da CEC nas complicações neurológicas, demonstrou que pacientes submetidos à cirurgia cardíaca com ou sem o auxílio da CEC, tiveram resultados semelhantes nos testes neuropsicológicos. Estes pacientes, avaliados previamente à cirurgia, foram submetidos a testes de perguntas e respostas simplificadas evocando a memória em dois momentos - na alta hospitalar e três meses depois - revelando que a CEC não foi a maior causa das disfunções neu- 
ropsicológicas. Outra especulação é a de que esses distúrbios neuropsicológicos estejam associados, em parte, a alterações transitórias da auto-regulação encefálica que acontecem por ação da ventilação artificial, anestésicos e variações da pressão arterial ${ }^{6}$.

Hoje sabe-se que as alterações cognitivas observadas no pós-operatório possuem causas multifatoriais. Aidade avançada, a presença de doença vascular difusa, a ocorrência de embolização no intra-operatório, de hipoperfusão e de inflamação conseqüente, a lesão por reperfusão, inerentes a este tipo de procedimento cirúrgico podem, em conjunto, determinar maior ou menor grau destes deficits cognitivos.

A formação de êmbolos, que tem como gênese ateromas da parede da aorta, agregados plaquetários, bolhas de ar oriundas do oxigenador e das câmaras cardíacas, podem sera causa primária de lesão encefálica ou do agravamento de lesões preexistentes. A microembolia gasosa é de difícil diagnóstico no intra-operatório e destaca-se como a principal geradora dos distúrbios cognitivos. Existem três causas principais para a origem das micro-bolhas. A primeira é a partir do próprio oxigenador; por ruptura do material da membrana, devido à pressão transmembrana elevada por fluxo excessivo de gases, com isto, pequenas quantidades de ar podem ganhar diretamente o sangue. A segunda causa é o processo de esfriamento e reaquecimento do sangue, que modifica as propriedades físicas dos gases (oxigênio, nitrogênio e gás carbônico), alterando-Ihes a solubilidade e predispondo a formação de microbolhas na corrente sangüínea. A terceira e última é que a abertura das câmaras cardíacas leva a entrada de ar para o interior dos vasos pulmonares e das cavidades cardíacas. Este ar, em pequenas quantidades, pode ganhar a circulação arterial e daí, diretamente, a circulação encefálica. Várias medidas têm sido propostas para contornar estes problemas. A principal está centrada no cirurgião, e consiste na retirada cuidadosa do ar das cavidades cardíacas e vasos pulmonares antes do despinçamento da aorta. Evitar os batimentos espontâneos do coração com a aorta aberta, antes de assegurar-se que todo o ar tenha sido removido, configura-se como outra importante conduta. Da parte do anestesiologista, a hiperventilação pulmonar, no momento da retirada do ar pelo cirurgião, pode determinar diminuição do fluxo sangüíneo encefálico. A diminuição do calibre dos vasos pela vasoconstrição pode tornar mais difícil a impactação dos microêmbolos, conforme mostram os trabalhos publicados por Linder e col. ${ }^{7}$.

O ateroembolismo aórtico também representa risco para os pacientes que se submetem à cirurgias cardíacas. Sabe-se que a doença ateromatosa aórtica agrava-se conforme a idade, sendo detectada em quase $80 \%$ dos pacientes a partir da oitava década. Indivíduos com doença vascular encefálica prévia ou acidente isquêmico transitório, portadores de doença ateromatosa comprovada nas artérias encefálicas extracranianas, possuem maior probabilidade de desenvolverem AVE no intra-operatório. Da mesma forma que a idade, o diabete melito e a hipertensão arterial são fatores independentes que podem influenciar nas complicações neurológicas. Assim, portadores destas afecções terão risco adicional durante a cirurgia cardíaca com CEC. Microembolização e alterações da perfusão encefálica devido à hipotensão arterial, freqüente neste tipo de operação, constituem outro agravante passível de provocar lesões neurológicas. Deve-se, também, considerar que a doença vascular aterosclerótica difusa, ao promover estreitamento do calibre dos vasos encefálicos, afeta sua auto-regulação que, frente às variações da pressão arterial e possível embolização, torna certas regiões mais susceptíveis à isquemia ${ }^{8}$.

Aresposta inflamatória, conforme apontado na figura 1, pode contribuir com a lesão encefálica, principalmente nas regiões que sofreram algum grau de hipoperfusão, fenômeno conhecido como lesão pós-reperfusão. Durante a CEC, a exposição do sangue à superfície não endotelial ativa a cascata da coagulação, o sistema fibrinolítico e o complemento, bem como favorecem a desgranulação leucocitária e a liberação de radicais livres. As regiões encefálicas, que eventualmente sofreram algum grau de hipoperfusão ou isquemia por microembolização, teriam maior probabilidade de agravamento das lesões pela ação da cascata da inflamação, que pode causar pequenas lesões com importante conseqüência clínica $^{8,9}$.

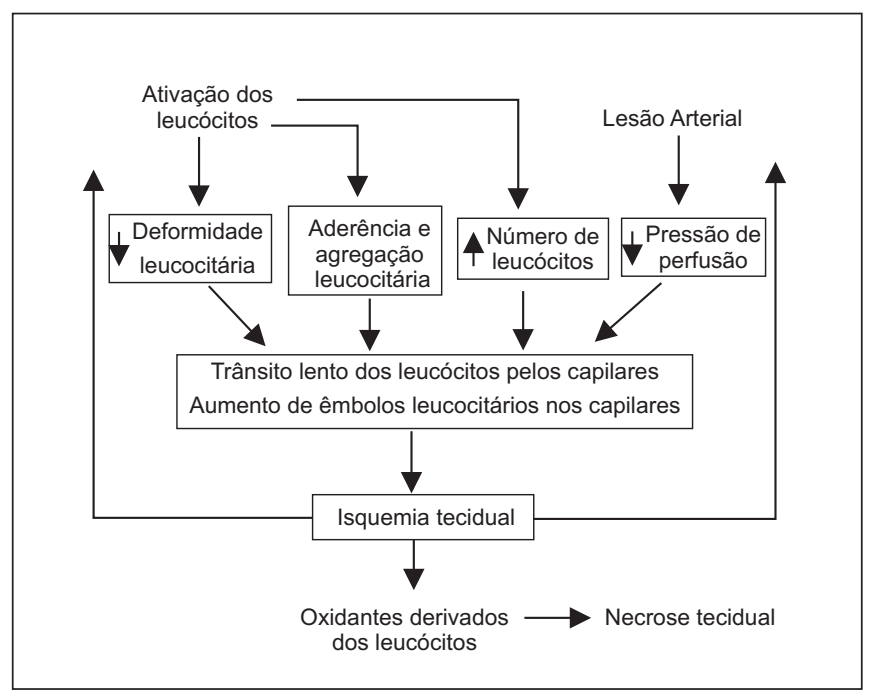
$\underset{\text { ria }^{8}}{\text { Figura }}{ }^{8}$ - Amplificação da Isquemia durante a Ativação Leucocitá-

Conforme visto, a própria cirurgia em si, somada à idade avançada dos pacientes submetidos à cirurgia cardíaca e aos outros fatores discutidos, já aumenta o risco de eles desenvolverem algum tipo de seqüela neurológica. É fato, também, que a maioria da população que se submete a esse tipo de procedimento cirúrgico, já apresenta uma série de componentes que podem aumentar os riscos de distúrbios neurológicos. Em vista disso, métodos têm sido propostos para que possam diminuir essas seqüelas e até a morbidade destes pacientes.

Dessa forma, Arrowsmith e col. ${ }^{9}$ classificaram as intervenções protetoras durante a cirurgia cardíaca em dois tipos: físicas e farmacológicas. As intervenções protetoras do tipo fí- 
sicas geralmente são empregadas no intra-operatório e incluem cirurgia rápida e eficaz, temperatura e PAM adequadas, controle da glicemia e $\mathrm{CO}_{2}$. A temperatura tem sido destaque dentre as intervenções físicas, sendo a hipotermia considerada por alguns uma ferramenta para a proteção encefálica. Dois estudos ${ }^{10,11}$, resumidos na tabela II, investigaram o efeito da temperatura durante a CEC. Em estudo feito por Martin e col. ${ }^{10}$, os pacientes submetidos à CEC foram divididos em dois grupos: no primeiro grupo a temperatura foi mantida a $35^{\circ} \mathrm{C}$ ou mais e no segundo grupo a temperatura foi mantida a $28^{\circ} \mathrm{C}$ ou menos. Os resultados obtidos indicaram que no grupo hipotérmico a incidência de complicações neurocognitivas foi três vezes menor do que no grupo aquecido ${ }^{10}$. Outro estudo com método semelhante conduzido por outro grupo de investigadores ${ }^{11}$ não conseguiu demonstrar resultados semelhantes ao anterior.

Tabela II - Conseqüências Neurológicas da Circulação Extracorpórea ${ }^{10,11}$

\begin{tabular}{llcc}
\hline & & $\begin{array}{c}\text { Hipotermia } \\
(\%)\end{array}$ & $\begin{array}{c}\text { Normotermia } \\
(\%)\end{array}$ \\
\hline Martin TD, Craver JM & $\begin{array}{l}\text { Acidente vascular } \\
\text { encefálico }\end{array}$ & 12 & 4,1 \\
Gott JP e col. & Encefalopatia & 0,2 & 0,4 \\
$(\mathrm{n}=1.001)$ & Total & 1,4 & 4,5 \\
$\begin{array}{l}\text { Warm Heart } \\
\begin{array}{l}\text { Investigators } \\
(\mathrm{n}=1.732)\end{array}\end{array}$ & $\begin{array}{l}\text { Acidente vascular } \\
\text { encefálico }\end{array}$ & 1,6 & 1,5 \\
\hline
\end{tabular}

O valor da pressão arterial média (PAM), durante a cirurgia também tem sido motivo de especulações científicas. Conforme sumarizado na tabela III, estudo feito por Gold e col. ${ }^{12}$ demonstraram que a pressão arterial (PA), mantida em valores superiores a $80 \mathrm{mmHg}$, está relacionada à menor incidência de lesões neurológicas. Estes autores compararam dois grupos, um, mantido em CEC com PAM entre 50 e $60 \mathrm{mmHg}$ e outro, com PAM igual ou maior que $80 \mathrm{mmHg}$. Os testes neurológicos a que estes pacientes foram submetidos no pós-operatório apontaram para um índice mais baixo de complicações neurológicas no grupo com PAM maior que $80 \mathrm{mmHg}$.

Tabela III - Efeito da PAM em Lesões Neurológicas, após Cirurgia Cardíaca, em Pacientes Separados por Baixa ou Alta Pressão durante a CEC ${ }^{12}$

\begin{tabular}{lccc}
\hline & $\begin{array}{c}\text { PAM } 50 \text { a 60 } \\
\mathrm{mmHg}(\%) \\
\mathrm{n}=124\end{array}$ & $\begin{array}{c}\text { PAM } 80 \text { a } 100 \\
\mathrm{mmHg}(\%) \\
\mathrm{n}=124\end{array}$ & $\mathrm{p}^{\mathrm{a}}$ \\
\hline Acidente vascular encefálico & 7,2 & 2,4 & 0,076 \\
Neurodeficiências menores & 3,2 & 0,8 & - \\
Complicações cardíacas & 4,8 & 2,4 & 0,03 \\
Deficiência cognitiva após & 12 & 11 & - \\
$6^{\circ}$ mês & 12,9 & 4,8 & 0,026 \\
$\begin{array}{l}\text { Complicação cardíaca maior e } \\
\text { disfunções neurológicas }\end{array}$ & 4 & 1,6 & 0,25 \\
Mortes & & &
\end{tabular}

Embora o resultado deste estudo não deixe qualquer dúvida sobre o valor mais elevado da PAM durante a cirurgia cardíaca, na menor incidência de complicações neurológicas, este fato deve ser reforçado principalmente em pacientes mais idosos, ou aqueles portadores de lesões obstrutivas da carótida e/ou história de hipertensão arterial grave. Deve ser ressaltado, também, o papel do gás carbônico $\left(\mathrm{PaCO}_{2}\right)$ e da glicemia. O primeiro, conforme já descrito, em níveis mais baixos teria algum papel na profilaxia da microembolização no momento de reiniciarem-se os batimentos cardíacos ${ }^{8}$. Quanto ao segundo, a hiperglicemia (valores acima de $120 \mathrm{mg} \%$ ) parece potencializar as lesões isquêmicas, envolvendo o metabolismo do cálcio pelas células durante o desenvolvimento da lesão de reperfusão. É consenso hoje a recomendação do rígido controle da glicemia pelos anestesiologistas durante a fase operatória ${ }^{8,9}$.

Quanto às intervenções farmacológicas, estas se propõema modular a atividade neuronal por meio de substâncias com comprovada ação, como inibidores da via de isquemia e/ou supressores metabólicos ${ }^{9}$. Entre estas substâncias tem-se dado atenção ao propofol, por seu efeito na supressão das espículas no eletroencefalograma (EEG). Levantou-se a provável questão se esse efeito poderia ter papel na redução do metabolismo encefálico durante a CEC agindo, portanto, como protetor neuronal. De acordo com Souter e col. ${ }^{13}$, o propofol, quando administrado nas doses que suprimem as ondas do EEG durante a CEC, não reduziu durante o reaquecimento a saturação venosa de oxigênio encefálico, abaixo de $50 \%$, medida no bulbo da veia jugular interna. Os inibidores da via de isquemia celular, como antagonistas de cálcio, estão sendo avaliados como possíveis protetores. Agentes antidepressivos poderiam estar contribuindo com uma nova estratégia dirigida para a inibição leucocitária, proteção endotelial, inibição de receptores aminoácidos excitatórios, entre outros, promovendo algum grau de proteção orgânica durante a CEC ${ }^{9}$. Entretanto, a comprovação clínica destes resultados ainda necessita de maior tempo de observação.

Mesmo utilizando métodos que possam surtir efeito protetor e com todos os cuidados tomados no pré, intra e pós-operatório, existem ainda pacientes que, submetidos a riscos semelhantes desenvolvem situações clínicas diferentes, levando a crer que há influência genética em cada caso. Atualmente, com o desenvolvimento dos laboratórios de pesquisa genética, está sendo possível identificar substâncias marcadoras da função neuronal. Estas substâncias estariam envolvidas com a proteção encefálica ou a maior tendência de lesão celular frente a uma agressão ou, até mesmo, ajudando para um rastreamento quanto a um diagnóstico ou prognóstico mais preciso. Em uma das diversas investigações realizadas nesse campo, voltou-se para a proteína $\mathbf{S 1 0 0 .}$ Essa proteína pode ser um marcador promissor da lesão neuronal. Normalmente, a proteína $\mathrm{S} 100$ não faz parte das substâncias presentes no plasma sangüíneo, porém seu surgimento no líquido plasmático acontece após um AVE, na hemorragia subaracnóidea, no trauma craniano e após a CEC ${ }^{14}$. Para tentar compreender a forma de liberação dessa proteína após a CEC, Johnson e col. ${ }^{15}$ observaram 515 pacien- 
tes submetidos à cirurgia de RM e mediram a concentração de S100 ao final da CEC, após 5, 15 e 48 horas, ao mesmo tempo em que realizavam acompanhamento clínico-neurológico. Os resultados obtidos mostraram que a liberação precoce de S100 estava relacionada com a idade e o maior tempo de CEC, não havendo correlação com o exame neurológico. Porém, a liberação tardia de $\mathrm{S} 100$ apresentou associação com o exame neurológico positivo e a incidência de complicações encefálicas. Apesar de a proteína $\mathrm{S} 100$ ser um possível indicador da gravidade da lesão encefálica, ela não é capaz de informar a extensão anatômica nem a conseqüência clínica dela. Enquanto as investigações aumentam a fim de encontrar substâncias capazes de auxiliar em um prognóstico melhor, surgem especulações sobre outras já bem conhecidas. Uma dessas substâncias é o glutamato, que poderia ser um possível marcador da lesão encefálica. Porém, Reynolds e col. ${ }^{16}$, com o intuito de saber se o glutamato se correlacionaria com a lesão neuronal, desenvolveram um método simples e confiável de quantificar sua concentração no plasma durante as cirurgias de RM com CEC. A variação da concentração de glutamato foi obtida durante a operação. Testes neurocognitivos foram aplicados nos pacientes que participavam da pesquisa, antes e após a cirurgia. Durante a cirurgia, observou-se que a concentração de glutamato variava de acordo com o tempo e conforme cada paciente. A partir das dosagens séricas de glutamato, durante a cirurgia e os testes neurológicos, Reynolds e col. concluíram que as variações da concentração de glutamato no plasma não eram preditivas para caracterizar os distúrbios neurológicos verificados após a cirurgia de RM ${ }^{16}$.

Nessa busca de possíveis substâncias preditivas da lesão encefálica, surgem outras que poderiam influenciar o prognóstico de cada paciente. E, nesse caso, as atenções têm-se voltado para a Apolipoproteína E (ApoE). Alguns estudos já indicam que ela pode estar envolvida no declínio cognitivo após a cirurgia cardíaca. Tem-se por certo que a presença de um dos alelos da ApoE é um importante fator de risco para a forma de início mais tardia do Mal de Alzheimer, além da provável associação no aumento de risco de aterosclerose. A ApoE é codificada geneticamente em seres humanos sob três isoformas designadas: ApoE $\varepsilon 2$, ApoE $\varepsilon 3$ e ApoE $\varepsilon 4{ }^{17}$.

Em estudo realizado ${ }^{18}$ em várias populações mostrou-se que a variação alélica da $A p o E ~ \& 4$ confere um aumento de risco para Mal de Alzheimer, enquanto o alelo $\varepsilon 2$ confere um efeito protetor. A hipótese levantada foi que o alelo $\varepsilon 4$ poderia estar envolvido na auto-regulação do fluxo sangüíneo encefálico. Em estudo realizado por Ti e col. ${ }^{19}$, compararam-se a auto-regulação do fluxo encefálico, a taxa de metabolismo encefálico de $\mathrm{O}_{2}$ e a diferença de concentrações de $\mathrm{O}_{2}$ no sangue arterial e venoso de pacientes com ou sem o alelo $\varepsilon 4$. Foram selecionados 154 pacientes que se submeteriam à cirurgia de RM. Durante a cirurgia, mediu-se o fluxo sangüíneo encefálico usando $\mathrm{Xe}^{133}$, e calculou-se a taxa de metabolismo encefálico pela diferença de concentração de $\mathrm{O}_{2}$ no sangue arterial e sangue venoso misto, obtido no bulbo jugular. A conclusão a que o grupo de Ti e col. chegou foi a de que o alelo $\varepsilon 4$ não tem efeito global sobre o fluxo, a oferta e a extração de

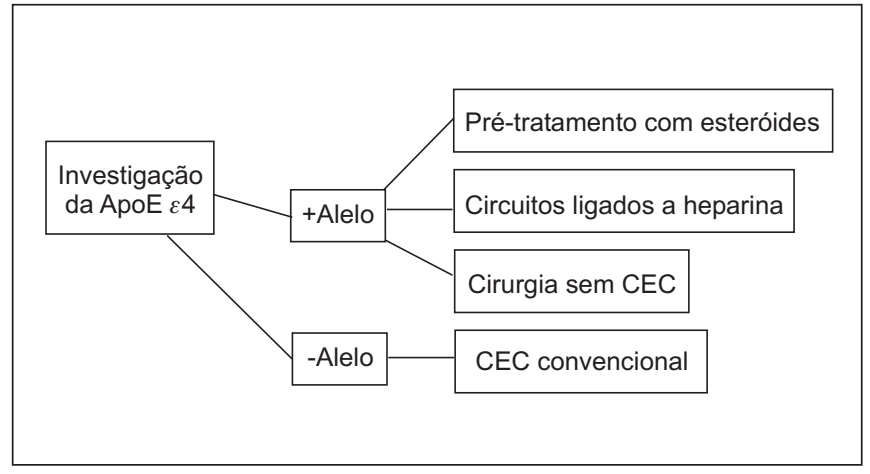

Figura 2 - Fluxograma de Genótipo ${ }^{17}$

$\mathrm{O}_{2}$, o que sugere que os efeitos do alelo $\varepsilon 4$ na lesão encefálica sejam devidos a outros mecanismos. Um dos mecanismos, ao qual o alelo $\varepsilon 4$ possa estar envolvido, é o aumento de substâncias que atuam na ativação da resposta inflamatória como Interleucina 8 (IL-8) e Fator de Necrose Tumoral Alfa $(T N F-\alpha)$. A partir dessa informação, Drabe e col. ${ }^{20}$ objetivaram comprovar a relação entre as citocinas e o alelo $\varepsilon 4$, depois de cirurgias de RM com CEC. O grupo de Drabe estudou 22 pacientes medindo a concentração de IL-8 e TNF- $\alpha$, pelo método de radioimunoensaio, em intervalos regulares de 48 horas após a cirurgia, ao mesmo tempo que determinavam o alelo da ApoE nos pacientes. Nos resultados obtidos, seis pacientes $(27 \%)$ apresentavam o alelo para $\varepsilon 4$. Comparando o tempo de CEC, o tempo de pinçamento da aorta e a concentração de citocinas (IL-8 e TNF- $\alpha$ ), determinou-se que houve diferença significativa para os $27 \%$ dos pacientes estudados, pois estes tiveram aumento nos parâmetros citados, concluindo que a presença do alelo $\varepsilon 4$ pode estar associada ao aumento de IL-8 e TNF- $\alpha$ na CEC.

\section{CONCLUSÃO}

Acirurgia cardíaca tem usufruído de muitos avanços tecnológicos nas últimas décadas, não apenas com o advento de novas técnicas anestésico-cirúrgicas, mas também com o desenvolvimento de diversos fármacos que, comprovadamente, reduzem a incidência de complicações cardiovasculares a curto e longo prazo. A despeito disso, observa-se que, aparentemente, mesmo diante de bons resultados nos seguimentos em longo prazo, algumas complicações emergem, sem que se consiga estabelecer, com razoável grau de certeza, qual evento predisponente ocasionou a morbidade. Adisfunção neurológica pós-operatória é importante causa de problemas nesse período, podendo variar desde desconfortos transitórios, como desorientação, deficit de atenção, até a danos irreversíveis do sistema nervoso central, como hemorragia e isquemia encefálica.

Sabe-se que existem alguns fatores de risco para o aparecimento de problemas relativos ao sistema nervoso central no pós-operatório de cirurgia cardíaca, como utilização de CEC, presença de doença ateromatosa grave na aorta ascendente e carótidas, anticoagulação inadequada durante a 
cirurgia com CEC, idade avançada, indução de alterações na temperatura corporal intra-operatória, hiperglicemia, métodos de correção ácido-base no intra-operatório, micro e macroembolização em CEC, procedimentos intracardíacos, doença vascular encefálica avançada e doença cardíaca prévia ao procedimento. Ainda assim, esses fatores de risco continuam sob investigação para avaliar qual é o seu real impacto sobre o prognóstico neurológico pós-operatório.

Apredisposição genética a diversas doenças tem sido extensamente estudada ultimamente. Um exemplo é o estudo que está sendo desenvolvido pelos autores em conjunto com o Laboratório de Genética do Instituto do Coração do Hospital das Clínicas, acompanhando pacientes que estão sujeitos a cirurgia eletiva de RM. Por meio de um exame neurológico simples, conhecido como mini-mental comparam-se os resultados deste à fenotipagem da ApoE, afim de detectar se os pacientes que possuem o fenótipo para ApoE $\varepsilon 4$ demonstram maior predisposição à complicações neurológicas pós-cirúrgicas. O teste de amostragem sugere um número expressivo de pacientes para que se possa chegar a algum resultado, ainda em fase inicial. Este exame mini-mental é composto de questionário com perguntas simples, procurando testar a memória recente e tardia dos pacientes antes, 48 horas e no momento da alta hospitalar. Havendo complicações mais sérias ou alteração do mini-mental, o neurologista é acionado para os exames especializados. A revelação de que alguns indivíduos possuem tendência hereditária para desenvolver certos estados mórbidos, permitirá que novas terapias, conforme delineados na figura 2 , sejam instituídas em fases bem mais precoces antecipando-se ao fator agressor, o que poderia proporcionar um significativo impacto sobre a qualidade de vida e sobre a morbimortalidade. Assim, faz-se necessário estabelecer, da forma mais correta possível, quais são os fatores predisponentes ao processo patogênico e os seus efeitos.

Portanto, o risco significativo de efeitos neurológicos adversos, durante a cirurgia cardíaca, explica o interesse renovado nas estratégias de proteção, na fisiopatologia das lesões e, atualmente, na provável genética envolvida nas principais injúrias do sistema nervoso central ${ }^{21}$.

\section{Pathophysiology of Neurological Injuries during Heart Surgery}

Rólison Gustavo Bravo Lelis, M.D.; José Otávio Costa Auler Júnior, TSA, M.D.

\section{INTRODUCTION}

Recent technological advances have led to decreased morbidity and mortality during heart surgeries. Neurological complications, however, are still important causes of postoperative morbidity and respond for great part of postoperative deaths.
Cardiopulmonary bypass (CPB) was introduced 50 years ago, and since then, reports have been published about patients developing some type of neurological sequela, fostering extensive researches on these sequela and CPB itself. It is believed that the overall incidence of such complication is up to $75 \%^{1}$, and may vary from transient psychological changes, such as attention and memory deficits, to more severe events, such as extensive stroke. It is currently known that such complications after heart surgery may involve different brain areas and present a variety of clinical manifestations.

Many factors play different roles on perioperative neurological injury origin during heart surgeries, like older age, previous brain or atheromatous disease, diabetes mellitus, intracardiac procedures, changes in body temperature during CPB and maybe a genetic predisposition, among others.

This review aimed at discussing major factors involved in heart surgery-related neurological injuries and their possible genetic origin, as well as proposing some measures for their prevention.

\section{INCIDENCE AND PATHOPHYSIOLOGY}

The literature shows a wide variation in the incidence of neurological complications after heart surgeries. Discrepancy of results is attributed to different study designs (whether prospective or retrospective, for example), the nature of the surgery (open or closed heart surgeries), the presence of co-morbidity and the method used to evaluate type and degree of neurological dysfunctions. Studies on postoperative neurological dysfunctions are primarily related to myocardial revascularization because it represents most heart surgeries. Although there are discrepancies regarding the methods, the incidence of neurological complications varies from $0.4 \%$ to $5.4 \% 1$

Even admitting that cardiopulmonary bypass is the major factor involved in neurological complications during heart surgeries, there have always been some questions about the potential noxious effect of the anesthetic-surgical procedure on the central nervous system. To verify this possibility, an European study ${ }^{2}$ has evaluated brain function of 1218 elderly patients submitted to non-cardiac surgeries. Neuro-cognitive tests through simple questionnaires with direct questions about name, birth date, current date, job, address, data on relatives, were applied in three periods: before surgery, at hospital discharge and three months later. There has been a relatively high incidence of postoperative cognitive dysfunction ( $26 \%)$, made evident by confusion of dates and facts, as well as by memory lapses persisting for up to three months in $10 \%$ of studied patients ${ }^{2}$. The study tried to attribute this cognitive dysfunction to the anesthetic-surgical procedure, in the absence of cardiopulmonary bypass. Shaw et al. ${ }^{3}$, following the same line of investigation and also employing neuro-cognitive tests based on simple questionnaires, have compared two groups of patients: one submitted to myocardial revascularization with cardiopulmonary bypass, and the other submitted to peripheral vascular surgery. Results are 
shown in table I and reveal higher incidence of cognitive dysfunction in the first group. What can be drawn from this and other investigations is that, for sure, cardiopulmonary bypass is a predisposing factor for cognitive dysfunctions, but that they are also present in non-cardiac surgeries, especially in patients with history of arterial vascular disease.

Table I - Neurological Complications during Heart Surgeries as Compared to Peripheral Vascular Surgeries ${ }^{3}$

\begin{tabular}{lcc}
\hline Deficit Severity & $\begin{array}{c}\text { Myocardial } \\
\text { Revascularization } \\
(\mathrm{n}=298)\end{array}$ & $\begin{array}{c}\text { Control } \\
(\mathrm{n}=48)\end{array}$ \\
\hline Mild (deficit in 1 or 2 tests) & $164(55 \%)$ & $15(31 \%)$ \\
Moderate (deficit in 3 or 4 tests) & $57(19 \%)$ & 0 \\
Severe (deficit in $\geq 5$ tests) & $14(4.7 \%)$ & 0 \\
\hline
\end{tabular}

The impact of cognitive changes on morbidity and mortality of patients submitted to heart surgery with cardiopulmonary bypass is not severe. The same is not true for more severe events, since injuries generically defined as strokes may lead to comma, permanent sequela or death. There has always been some confusion in the literature regarding names, classification and pathophysiology of more severe neurological events. It was up to a group of investigators led by Roach et al. ${ }^{4}$, studying a large series of patients, to propose a classification shown in chart I. Using the classification proposed by these investigators ${ }^{4}$, Wolman et al. ${ }^{5}$ have evaluated a group of patients submitted to myocardial revascularization and have classified neurological events in: type I, defined as focal injuries with stupor or comma; and type II, with intellectual function deterioration, memory deficits or seizures ${ }^{5}$.

Chart I - Proposal for Neurological Events Classification ${ }^{5}$ Brain death, non-fatal strokes and transient ischemic attacks (predisposing factors: aortic atherosclerosis, previous neurological disease, age)

Decreased intellectual capacity (predisposing factors: age, arType II terial hypertension, lung disease and excessive alcohol consumption)

This study has shown that $6.1 \%$ of patients had some neurological complication, being $3.1 \%$ type I, and $3 \%$ type $\mathrm{II}^{5}$. Still in this investigation, Wolman et al. ${ }^{5}$ have shown that, parallel to the surgical technique, age would also be a risk factor for neurological complications. Patients aged 70 years or above had a mean incidence of $4 \%$ to $9 \%$ postoperative stroke or comma, differently from patients below 70 years of age, who had an incidence of $1 \%$.

Other mechanisms to explain these complications have also been exhaustively studied. CPB itself has been considered the major cause for more severe postoperative cognitive changes. However, a study by Taggart et al. ${ }^{6}$ to establish the real impact of CPB on neurological complications has shown that patients submitted to heart surgeries with or without CPB had similar neuro-psychological test results. These patients, who had been evaluated before surgery, were submitted to simple questions and answers tests evoking memory in two moments: hospital discharge and three months later. Results have shown that CPB was not the major cause of neuro-psychological dysfunctions. Another speculation would be that such neuro-psychological disorders are partially associated to transient changes in brain auto-regulation as a consequence of artificial ventilation, anesthetic drugs and blood pressure changes ${ }^{6}$.

Today it is known that postoperative cognitive changes are multifactorial. Older age, diffuse vascular disease, intraoperative embolization, hypoperfusion and consequent inflammation, as well as reperfusion injury inherent to this type of procedure may, together, determine more or less cognitive deficits.

Embolus formation, caused by aortic wall atheromas, platelets aggregation and air bubbles from the oxygenator and cardiac chambers, may be the primary cause of brain injury or worsening of preexisting injuries. Intraoperative gas microembolism is difficult to detect and is considered the major cause for cognitive disorders. There are three main mechanisms for micro-bubbles formation. The first one is related to the oxygenator itself, when membrane material may rupture due to increased transmembrane pressure by excessive gas flow. This way, small amounts of air may directly reach the blood stream. The second cause is blood cooling and heating, which can modify gases' physical properties (oxygen, nitrogen and carbon dioxide). Solubility changes may predispose to micro-bubbles formation. Finally, cardiac chambers opening leads to air entrance into them and pulmonary vessels as well. This air, in small amounts, may get to arterial circulation and reach brain circulation next. Several measures have been proposed to overcome this problem. The most important is focused on the surgeon, and consists of careful air removal from cardiac cavities and pulmonary vessels before aortic unclamping. Preventing spontaneous heartbeats with open aorta before being sure that all air has been removed is also an important approach. For the anesthesiologist, pulmonary hyperventilation when air is being removed by the surgeon may determine decreased brain blood flow. Vessels caliber decreasing by vasoconstriction may reduce micro-emboli impactation, as shown by Linder et al. ${ }^{7}$.

Aortic atheroembolism is also a risk for patients submitted to heart surgeries. It is known that severe aortic atheromatous disease is worsened with age, being detected up to $80 \%$ of patients aged 80 years or above. Patients with previous cerebral vascular disease or transient ischemic attack, with proven atheromatous disease in extracranial brain arteries, have a higher chance of developing intraoperative stroke. Similar to age, diabetes mellitus and hypertension are independent factors which may affect neurological complications. So, patients with these diseases are at additional risk during heart surgery with CPB. Microembolization and brain perfusion changes due to arterial hypotension, which is frequent in this type of surgery, are other aggravating factors able to cause neurological injuries. It should also be consid- 
ered that diffuse atherosclerotic disease, promoting brain vessels narrowing, affects its auto-regulation which, in face of blood pressure changes and possible embolization, makes some brain areas more susceptible to ischemia ${ }^{8}$. Inflammatory response, as shown in figure 1, may contribute to brain injury, especially in brain areas with bad perfusion, a mechanism known as post-reperfusion injury. During CPB, blood exposure to non-endothelial surfaces activates the coagulation cascade, the fibrinolytic and complement systems, being able to induce leukocytes degranulation and free radicals release as well. Brain regions which might have suffered some degree of microembolization-induced hypoperfusion or ischemia would have a higher chance of worsening the injuries due to overimposed inflammatory events, with significant clinical consequences ${ }^{8,9}$.

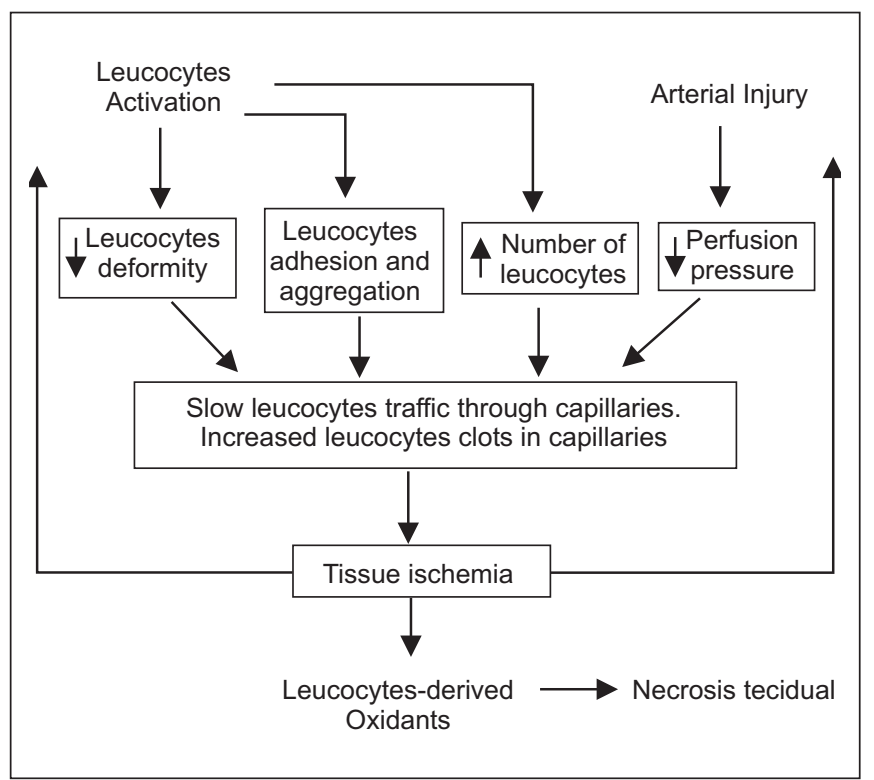

Figure 1 - Ischemia Amplification after Leukocytes Activation ${ }^{8}$

As seen, the surgery itself, in addition to older age of patients submitted to heart surgeries and other factors above discussed, increases the risk for some type of neurological sequela. It is also a fact that most candidates to this type of surgical procedure already present with several conditions which may increase the risks for neurological disorders. Therefore, many methods have been proposed to reduce such sequela and patients' morbidity.

This way, Arrowsmisth et al. ${ }^{9}$ have classified protective measures during heart surgeries in two types: physical and pharmacological. Physical protective measures are mainly related to the intraoperative period, and include fast and effective surgery, adequate temperature and MBP, as well as glycemia and $\mathrm{CO}_{2}$ control. Temperature management has been highlighted among physical measures, being hypothermia considered by some authors a useful tool for brain protection. Two studies ${ }^{10,11}$, summarized in Table II, have investigated the effect of temperature during CPB. In a study of Mar- tin et al. ${ }^{10}$, patients submitted to CPB were divided in two groups: in the first group, temperature was maintained at 35 ${ }^{\circ} \mathrm{C}$ or above, and in the second, temperature was maintained at $28{ }^{\circ} \mathrm{C}$ or below. Results have indicated that, in the hypothermal group, the incidence of neuro-cognitive complications was three times lower as compared to the warmer group ${ }^{10}$. Another similar study of a different group of investigators ${ }^{11}$ was unable to show similar results.

Table II - Neurological Consequences of Cardiopulmonary Bypass ${ }^{10,11}$

\begin{tabular}{llcc}
\hline & & $\begin{array}{c}\text { Hypothermia } \\
(\%)\end{array}$ & $\begin{array}{c}\text { Normothermia } \\
(\%)\end{array}$ \\
\hline Martin TD, Craver JM & Stroke & 12 & 4.1 \\
Gott JP et al. & Encephalopathy & 0.2 & 0.4 \\
$(\mathrm{n}=1.001)$ & Total & 1.4 & 4.5 \\
$\begin{array}{l}\text { Warm Heart } \\
\begin{array}{l}\text { Investigators } \\
(\mathrm{n}=1.732)\end{array}\end{array}$ & Stroke & 1.6 & 1.5 \\
\hline
\end{tabular}

Mean blood pressure (MBP) during surgery has also been an issue for scientific speculations. As summarized in table III, a study of Gold et al. ${ }^{12}$ has shown that mean blood pressure maintained above $80 \mathrm{mmHg}$ is related to a lower incidence of neurological injuries. The authors have compared two groups of patients: one of them with MBP kept between 50 and $60 \mathrm{mmHg}$ during $\mathrm{CPB}$, and the other with MBP equal to or above $80 \mathrm{mmHg}$. Postoperative neurological tests have shown a lower rate of neurological complications in the group with MBP above $80 \mathrm{mmHg}$.

Table III - Effects of MBP on Post Heart Surgery Neurological Injuries in Patients Divided in Low or High Blood Pressure during $\mathrm{CPB}^{12}$

\begin{tabular}{lccc}
\hline & $\begin{array}{c}\text { MBP 50 to 60 } \\
\mathrm{mmHg}(\%) \\
\mathrm{n}=124\end{array}$ & $\begin{array}{c}\mathrm{MBP} 80 \text { to 100 } \\
\mathrm{mmHg}(\%) \\
\mathrm{n}=124\end{array}$ & $\mathrm{p}^{\mathrm{a}}$ \\
\hline Stroke & 7.2 & 2.4 & 0.076 \\
Minor neurodeficiencies & 3.2 & 0.8 & - \\
Heart complications & 4.8 & 2.4 & 0.03 \\
$\begin{array}{l}\text { Cognitive deficiency after } \\
6^{\text {th }} \text { months }\end{array}$ & 12 & 11 & - \\
$\begin{array}{l}\text { Major heart complication and } \\
\text { neurological dysfunctions }\end{array}$ & 12.9 & 4.8 & 0.026 \\
Deaths & 4 & & 1.6 \\
\hline
\end{tabular}

Although this study's results leave no doubt about the value of higher MBP during heart surgery to decrease neurological complications, one should keep in mind that this is particularly true for older patients or those with carotid obstructive injuries and/or history of severe hypertension. It should also be highlighted the role of carbon dioxide $\left(\mathrm{PaCO}_{2}\right)$ and glycemia. As mentioned above, lower $\mathrm{PaCO}_{2}$ levels would play some role in microembolization prevention when heartbeats are restarted Vol. 54, No 4, Julho - Agosto, 2004 
${ }^{8}$. Also, glycemia levels above $120 \mathrm{mg} \%$ seem to potentiate ischemic injuries, a mechanism which involves cell calcium metabolism during reperfusion injury development. Hence, the strict control of glycemia by anesthesiologists in the intraoperative period is a basic recommendation today ${ }^{8,9}$. Pharmacological measures aim at modulating neuronal activity by means of substances of proven action, such as ischemic pathway inhibitors and/or metabolic suppressors ${ }^{9}$. Among these substances, special attention has been given to propofol for its effect on EEG spikes suppression. A question has been raised whether such effect could play a role in decreasing brain metabolism during $\mathrm{CPB}$, thus acting as neuronal protector. According to Souter et al. ${ }^{13}$, propofol in doses suppressing EEG waves during CPB has not decreased brain venous oxygen saturation below $50 \%$ during reheating, measured in internal jugular vein bulb.

Cell ischemia pathway inhibitors, such as calcium antagonists are being evaluated as potential protectors. Antidepressant agents could aid for a new strategy aiming at leukocytes inhibition, endothelial protection and excitatory aminoacid receptors inhibition, among others, promoting some degree of organic protection during $C \mathrm{~PB}^{9}$. However, clinical confirmation of such results still requires further investigations.

Even with protective methods and the proper care during pre, intra and postoperative periods, there are still patients who, submitted to similar risks, present different clinical evolutions, making us believe in a case-by-case genetic influence. Currently, with the development of genetic research labs, it is possible to identify neuronal function markers. These markers would be involved in brain protection or a higher trend for cell injury after an aggression, or would even help patient's screening for more accurate prognosis or diagnosis. One of the several investigations in this area has targeted $\mathrm{S} 100$ protein, which may be a promising neuronal injury marker. S100 protein is not an usual part of blood plasma substances, but it is present in plasma after stroke, subarachnoid hemorrhage, brain trauma and $\mathrm{CPB}{ }^{14}$. Trying to understand how this protein is released after CPB, Johnson et al. ${ }^{15}$ have observed 515 patients submitted to MR and have monitored S100 concentration at CPB completion, after 5, 15 and 48 hours, together with clinical-neurological follow-up. Data have shown that early S100 release was related to age and longer CPB time, with no correlation with neurological tests. Late S100 release, however, was associated to positive neurological tests and the incidence of brain injuries. Although being a possible indicator of brain injury severity, S100 protein is unable to inform its anatomic extension or clinical consequences. While investigations progress toward finding effective substances to improve diagnosis, there are speculations about other already well-known substances, among them glutamate, which could be a brain injury marker. Reynolds et al. ${ }^{16}$, however, in an attempt to know whether glutamate would correlate to neuronal injuries, have developed a simple and reliable method to quantify its plasma concentration during MR with CPB. Glutamate concentration changes were obtained during surgery. Neuro-cognitive tests were applied before and after surgery. It has been observed that glutamate concentration during surgery would vary according to each patient and with time. Based on intraoperative serum glutamate concentrations and neurological tests, Reynolds et al. have concluded that plasma glutamate changes were not predictive of neurological disorders observed after $\mathrm{MR}^{16}$.

In the search for brain injury predictors, others substances were identified which could influence prognosis. Toward this goal, attentions were turned to apoliprotein E (ApoE). Some studies already indicate that it could be involved with cognitive decrease after heart surgery. It is certain that the presence of one of the ApoE's alleles is an important risk factor for late onset Alzheimer's disease, in addition to its potential association with increased risk for atherosclerosis. ApoE is genetically coded in men under three isoforms: ApoE $\varepsilon 2, A p o E$ $\varepsilon 3$ and ApoE $\varepsilon 4{ }^{17}$. A multi populational study ${ }^{18}$ has shown that ApoE $\varepsilon 4$ allelic variation increases the risk for Alzheimer's disease, while $\varepsilon 2$ allele has a protective effect. It has been postulated that allele $\varepsilon 4$ could be involved in brain blood flow auto-regulation. A study by $\mathrm{Ti}$ et al. ${ }^{19}$ has compared brain blood flow auto-regulation, brain $\mathrm{O}_{2}$ metabolic rate and arterial/venous difference in blood $\mathrm{O}_{2}$ concentrations in 154 patients with or without allele $\varepsilon 4$ submitted to MR. Brain blood flow was measured during surgery using $\mathrm{Xe}^{133}$ and brain metabolic rate was calculated by the difference between $\mathrm{O}_{2}$ concentrations in arterial and mixed venous blood obtained in the jugular bulb. The conclusion was that allele $\varepsilon 4$ had no global effect on brain blood flow, $\mathrm{O}_{2}$ supply and extraction, suggesting that allele $\varepsilon 4$ effects on brain injuries are due to different mechanisms. One of them could be increasing substances which act on inflammatory response activation, such as Interleukin 8 (IL-8) and Tumor Necrosis Factor Alpha $(T N F-\alpha)$. With that in mind, Drabe et al. ${ }^{20}$ have decided to verify the possible relationship between cytokines and the allele $\varepsilon 4$ after MR with CPB. Drabe's group has studied 22 patients by monitoring IL- 8 and TNF- $\alpha$ concentrations thru radioimmunoassay in 48-hour intervals after surgery, at the same time that they determined the presence of ApoE $\varepsilon 4$ allele. Their results have shown 6 patients $(27 \%)$ with this allele. When comparing CPB time, aortic clamping time and cytokines concentration (IL=8 and TNF- $\alpha$ ), there has been significant difference for these $27 \%$ of patients as they had those parameters increased. The conclusion was that the presence of allele $\varepsilon 4$ could be associated to increased IL- 8 and TNF- $\alpha$ during CPB.

\section{CONCLUSION}

Heart surgery has experienced major technological advances in recent years, not only with the advent of new anesthetic-surgical techniques, but also with the development of different drugs, which knowingly decrease the incidence of short and long-term cardiovascular complications. Nevertheless, it has been observed that, even with good results in long-term follow-ups, there are still some complications for which it is not possible to determine, with a reasonable level of certainty, the predisposing event leading to morbidity. Post- 
operative neurological dysfunction is an important source of problems, which may vary from transient distress such as disorientation or attention deficits, to irreversible central nervous system injuries, such as brain hemorrhage and ischemia.

It is known that there are some risk factors for central nervous system related problems in the postoperative period of heart surgeries, such as CPB, severe ascending aorta and carotid atheromatous disease, inadequate anticoagulation during surgery with $\mathrm{CPB}$, older age, induction of intraoperative body temperature changes, hyperglycemia, intraoperative acid-base correction methods, micro and macroembolization in $\mathrm{CPB}$, intracardiac procedures, advanced cerebrovascular disease and previous cardiac disease. Even so, these risk factors are still under investigation to determine their actual impact on postoperative neurological prognosis. Genetic predisposition to many diseases has been extensively studied. As an example, we have been developing a study together with the Genetic Laboratory of the Heart Institute, Hospital das Clínicas, where patients undergoing MR are being followed-up. Results of a simple neurological evaluation, known as mini-mental, are compared to ApoE phenotyping to detect whether patients with phenotype for ApoE $\varepsilon 4$ are prone to postoperative neurological complications. Sampling test suggests that an expressive number of patients is needed to achieve any result, still in the early stage of the study. This mini-mental evaluation is composed of a questionnaire with simple questions to test recent and late memory, before, at 48 hours and at hospital discharge. If more severe complications or mini-mental changes are detected, the neurologist is called for specialized tests. The confirmation that some individuals present hereditary trends to develop some morbid states will allow new therapies to be earlier instituted, as shown in figure 2, before the aggressing factor, what could have a significant impact on life quality and morbidity/mortality. Therefore, it is necessary to establish, as accurately as possible, which are the factors predisposing to pathogenic processes and their effects.

The significant risk of adverse neurological events during heart surgery explains the renewed interest on protective strategies, injuries pathophysiology and, more recently, on

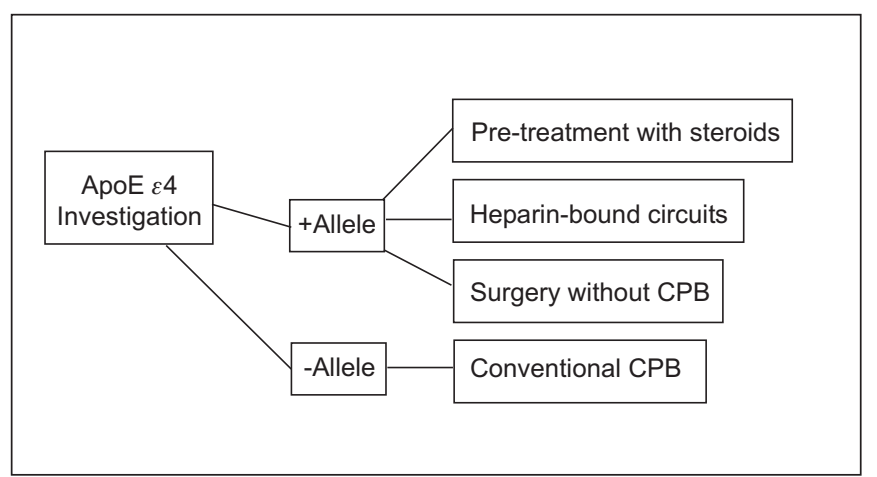

Figure 2 - Genotype Flowchart the genetics likely to be involved in main central nervous system injuries ${ }^{21}$.

\section{REFERÊNCIAS - REFERENCES}

01. Mangano DT - Cardiovascular morbidity and CABG surgery - a perspective: epidemiology, costs, and potential therapeutic solutions. J Card Surg, 1995;10:(Suppl4):366-368.

02. Moller JT, Cluitmans P, Rasmussen LS et al - Long term postoperative cognitive dysfunction in the elderly ISPOCD1 study. ISPOCD investigators. International study of postoperative cognitive dysfunction. Lancet, 1998;351(9106):857-861.

03. Shaw PJ, Bates Figure 2 - Genotype FlowchartD, Cartlidge NE et al - Neurologic and neuropsychological morbidity following major surgery: comparison of coronary artery bypass and peripheral vascular surgery. Stroke, 1987;18:700-707.

04. Roach GW, Kanchuger M, Mangano CM et al - Adverse cerebral outcomes after coronary bypass surgery. Multicenter study of perioperative ischemia research group and the ischemia research and Education Foundation Investigators. N Engl J Med, 1996;335:1857-1863.

05. Wolman RL, Nussmeier NA, Aggarwal A et al - Cerebral injury after cardiac surgery: identification of a group at extraordinary risk. Multicenter study of perioperative ischemia research group (McSPI) and the ischemia research Education Foundation (IREF) Investigators. Stroke, 1999;30:514-522.

06. Taggart DP, Browne SM, Halligan PW et al. - Is cardiopulmonary bypass still the cause of cognitive dysfunction after cardiac operations? J Thorac Cardiovasc Surg, 1999;118:414-421.

07. van der Linden J, Casimir-Ahn H - When do cerebral emboli appear during open heart operations? A transcranial Doppler study. Ann Thorac Surg, 1991;51:237-241.

08. Cook DJ - Neurologic Effects, em: Gravllee PG, Davis FR, Kursz $\mathrm{M}$ et al - Cardiopulmonary Bypass: Principle and Practice, $2^{\text {nd }}$ Ed, Philadelphia, Lippincott Williams \& Wilkins, 2000;403-431.

09. Arrowsmith JE, Grocott HP, Reves JG et al - Central nervous system complications of cardiac surgery. $\mathrm{Br} \mathrm{J}$ Anaesth, 2000;84:378-393.

10. Martin TD, Craver JM, Gott JP et al - Prospective, randomized trial of retrograde warm blood cardioplegia: myocardial benefit and neurologic threat. Ann Thorac Surg, 1994;57:298-304.

11. The Warm Heart Investigators - Randomized trial of normothermic versus hypothermic coronary bypass surgery. Lancet, 1994;343:(8897):559-563.

12. Gold JP, Charlson ME, Williams-Russo P et al - Improvement of outcomes after coronary artery bypass. A randomized trial comparing intraoperative high versus low mean arterial pressure. J Thorac Cardiovasc Surg, 1995;110:1302-1314.

13. Souter MJ, Andrews PJ, Alston RP - Propofol does not ameliorate cerebral venous oxyhemoglobin desaturation during hypothermic cardiopulmonary bypass. Anesth Analg, 1998;86:926-931.

14. Zimmer DB, Cornwall EH, Landar A et al - The S100 protein family: history, function, and expression. Brain Res Bull, 1995;37:417-429.

15. Jönsson $H$, Johnsson $P$, Alling $C$ et al - Significance of serum $\mathrm{S} 100$ release after coronary artery bypass grafting. Ann Thorac Surg, 1998;65:1639-1644.

16. Reynolds JD, Amory DW, Grocott HP et al - Change in plasma glutamate concentration during cardiac surgery is a poor predictor of cognitive outcome. J Cardiothorac Vasc Anesth, 2002;16:431-436. 
17. Nevin M - Neuropsychometric deficit after cardiac surgery: a new approach for a new millennium. $\mathrm{Br} J$ Anaesth, 2000;84:304-307.

18. Jorde LB, Carey JC, Bamshad MJ et al - Medical Genetics, $2^{\text {nd }}$ Ed, St. Louis, Mosby, 1999;231-232.

19. Ti LK, Mathew JP, Mackensen GB et al - Effect of apolipoprotein E genotype on cerebral autoregulation during cardiopulmonary bypass. Stroke, 2001;32:1514-1519.

20. Drabe N, Zund G, Grunenfelder J et al - Genetic predisposition in patients undergoing cardiopulmonary bypass surgery is associated with an increase of inflammatory cytokines. Eur $\mathrm{J}$ Cardiothorac Surg, 2001;20:609-613.

21. Auler Jr JOC - Neurologic Complications in Cardiac Surgery. Is Predictable? em: Gullo A- Anaesthesia Pain Intensive Care and Emergency: A.P.I.C.E. Trieste, Springer-Verlag, 2002;983-990.

\section{RESUMEN}

Lelis RGB, Auler Jr JOC - Lesión Neurológica en Cirugía Cardíaca: Aspectos Fisiopatológicos

JUSTIFICATIVA Y OBJETIVOS: En virtud de las lesiones neurológicas que ocupan un papel importante en el contexto de las complicaciones pós-operatorias, cuando comparadas a las demás, el presente estudio tentó discutir los principales factores envueltos en la lesión neurológica peri-operatoria en cirugía cardíaca, las intervenciones que buscan disminuir la incidencia de lesiones neurológicas, enfocando de manera simple, y la probable génesis genética en tales lesiones cerebrales.

CONTENIDO: Este trabajo contiene la revisión de artículos que enfocan la fisiopatología de las lesiones neurológicas relacionadas a procedimientos cardíacos, su posible origen genético, bien como una propuesta para su prevención.

CONCLUSIONES: Mucho se habló de las disfunciones cognitivas de pacientes después de cirugía cardíaca, como la revascularización del miocardio que es motivo del aumento de la morbimortalidad observada actualmente y factor de mayor tiempo de hospitalización. Es un hecho que ya hubo grandes avanzos en esa área con la finalidad de disminuir eses índices, previniendo aún otros que visan la profilaxis de lesiones neurológicas. 